\title{
A combination of differential equations and convolution in understanding the spread of an epidemic
}

\author{
ARNI S R SRINIVASA RAO ${ }^{1}$ and MASAYUKI KAKEHASHI ${ }^{2}$ \\ ${ }^{1}$ Centre for Ecological Sciences, Indian Institute of Science, Bangalore 560012 , \\ India \\ ${ }^{2}$ Institute of Health Sciences, Faculty of Medicine, Hiroshima University, \\ Hiroshima, 734 8551, Japan \\ e-mail: arni.srs.rao@ces.iisc.ernet.in
}

MS received 28 April 2003; revised 15 December 2003

\begin{abstract}
Nonlinear dynamical method of projecting the transmission of an epidemic is accurate if the input parameters and initial value variables are reliable. Here, such a model is proposed for predicting an epidemic. A method to supplement two variables and two parameters for this proposed model is demonstrated through a robust statistical approach. The method described here worked well in case of three continuous distributions. Model predictions could be lower estimates due to under-reporting of disease cases. An ad hoc procedure with a technical note is provided in the appendix.
\end{abstract}

Keywords. Dynamical model; likelihood; convolution; HIV.

\section{Introduction}

For the past several decades mathematical principles have had an important role in diseasecontrol strategies (Murray 1989; Anderson \& May 1991) and will continue to do so in future. Application of the models becomes easier if there are sufficiently accurate data for the variables used and the concerned parameter values. However, even a theoretical model developed with proper spread mechanisms without the use of any real data can assist in generating the required public health information. In this paper, a model is proposed for predicting an epidemic, where some of the variables and parameters in the model are obtained from convolution of statistical functions and likelihood estimation. This method is applicable when information about the early stages of the epidemic is not available, but appropriate surveillance reports are available for the later period. The method elaborated here is for HIV spread and is restricted to transmission dynamics of sexual infection between males and females. However, it can be extended to other infectious diseases as well. The treatment is mostly mathematical and does not go into details of the biology of epidemics. We demonstrate the method of predicting an epidemic and also show numerical results.

An earlier version of this paper was presented at the annual conference of the Indian Society for Mathematical Modelling \& Computer Simulation, Bangalore, November 14-15, 2002 


\section{Model}

Denote the populations of those not infected by HIV as $X_{0}, Y_{0}$, those infected as $X_{1}, Y_{1}$ and those who subsequently develop AIDS as $X_{2}, Y_{2}$ for males and females. Here, it is assumed that HIV-infected individuals will clinically progress to AIDS (the advanced stage of HIV infection) with an average incubation period $1 / \eta_{1}$ (for males) and $1 / \eta_{2}$ (for females). Infection is assumed to transmit from male to females and vice versa. No other mode of transmission is considered in the model. However, we discuss the applicability of the model to other modes of transmission in the conclusions section. Females transmit the virus to their male counterparts with a probability of $\beta_{01}$ (at contact rate $C$ ), while males transmit to females with a probability $\beta_{01}^{\prime}$ (at contact rate $C^{\prime}$ ). Males and females enter the susceptible class (susceptible here means those who can get the infection but are not infected yet) at exponential rates $\lambda_{1}$ and $\lambda_{2}$ i.e. $\lambda_{1(2)}(t)=\lambda_{0} e^{r_{1(2)} t}$. This assumption is very valid in the case of large population sizes. Infected individuals are allowed to withdraw from risk behaviour (at rates $\omega_{1}$ and $\omega_{2}$, say) and die naturally (at rates $\mu_{1}$ and $\mu_{2}$, say). These death rates are unrelated to AIDS, hence the same rates are applicable for non-infected individuals. Deaths which are due to AIDS are assumed to be the same (at rate $\mu$, say) for both the sexes, because on an average it is about 1 year from the development of AIDS. Now we form the the differential equations based on the above explanation for the transmission between males and females as follows:

$$
\begin{aligned}
\mathrm{d} X_{0} / \mathrm{d} t & =\lambda_{1}-\beta_{01} C X_{0} Y_{1}-\mu_{1} X_{0}, \\
\mathrm{~d} X_{1} / \mathrm{d} t & =\beta_{01} C X_{0} Y_{1}-\left(\omega_{1}+\eta_{1}+\mu_{1}\right) X_{1}, \\
\mathrm{~d} X_{2} / \mathrm{d} t & =\eta_{1} X_{1}-\mu X_{2}, \\
\mathrm{~d} Y_{0} / \mathrm{d} t & =\lambda_{2}-\beta^{\prime}{ }_{01} C^{\prime} Y_{0} X_{1}-\mu_{2} Y_{0}, \\
\mathrm{~d} Y_{1} / \mathrm{d} t & =\beta_{01}^{\prime} C^{\prime} Y_{0} X_{1}-\left(\omega_{2}+\eta_{2}+\mu_{2}\right) Y_{1}, \\
\mathrm{~d} Y_{2} / \mathrm{d} t & =\eta_{2} Y_{1}-\mu Y_{2} .
\end{aligned}
$$

Application of this model to explain the transmission dynamics from the beginning of the epidemic is less appropriate than from the recent past or the present situation as epidemiological parameters (viz, $\mu, \eta_{1}$ and $\eta_{2}$ ). More importantly, population sizes which can transmit (viz, $X_{1}, Y_{1}$ ) the virus to susceptible population are generally not available at the beginning. This is due to one or a combination of several factors like: low technology public health system, poor awareness (including misconceptions), social stigma, economic instability etc. When the population sizes at risk are used as multiplying factors, it is essential to know them accurately to provide valid predictions for the future. We explain a method in the next section for the estimation of $X_{1}$ and $Y_{1}$ for the initial input values for the model equations explained above. We also explain how obtain to the average incubation periods i.e. $1 / \eta_{1}$ and $1 / \eta_{2}$. We give numerical example for the statistical method of estimation and implementation of the above model in $\S 4$.

\section{Formation of the likelihood equation and estimation}

Let $R_{1}, R_{2}, R_{3} \ldots R_{N}$ be the number of AIDS cases reported in the calender time intervals $\left[\Omega_{0}, \Omega_{1}\right),\left[\Omega_{1}, \Omega_{2}\right),\left[\Omega_{2}, \Omega_{3}\right), \ldots\left[\Omega_{N-1}, \Omega_{N}\right)$ and $\vartheta$ be the HIV density that represents the past infection growth. The length of the incubation period $U$ and the time of HIV infection $V$ 
are independent random variables. The time of AIDS diagnosis $\Lambda=V+U$ (because since the time of HIV infection, AIDS develops after a length of time called incubation period). Now the distribution of cumulative AIDS is represented as a convolution of the two functions $\vartheta$ and $U$ as given below,

$$
N\left(\Omega_{0} \leq \epsilon<\Omega_{N}\right)=\Lambda\left(\Theta_{1}, \Theta_{2} \mid \Omega_{N}\right)=\int_{\Omega_{0}}^{\Omega_{N}} \vartheta\left(\epsilon \mid \Theta_{1}\right) U\left(\Omega_{N}-\epsilon \mid \Theta_{2}\right) \mathrm{d} \epsilon .
$$

Then through deconvoluting (7), we estimate parameters of incubation period to AIDS distribution (which is also called incubation distribution). An individual who is diagnosed at calendar time $\Omega_{N}$ must have got the infection at time prior to $\epsilon . \Theta_{1}$ and $\Theta_{2}$ are parameter(s) for the infection density and incubation distribution respectively. A similar equation was used for the first time to project AIDS cases in US (Brookmeyer \& Gail 1988). The conditional probability $p_{i}$ for an infected individual at time $V$ to be diagnosed with full-blown symptoms of AIDS before $\Omega_{N}$, which is when they actually develop it in the time interval $\left[\Omega_{j-1}, \Omega_{j}\right)$, is

$$
\begin{aligned}
p_{i} & =N\left(\Omega_{i-1} \leq \epsilon<\Omega_{i} / \Omega_{0} \leq \epsilon<\Omega_{N}\right) \\
& =\frac{N\left(\Omega_{0} \leq \epsilon<\Omega_{i}\right)-N\left(\Omega_{0} \leq \epsilon<\Omega_{i-1}\right)}{N\left(\Omega_{0} \leq \epsilon<\Omega_{N}\right)} \\
& =\frac{\Lambda\left(\Theta_{1}, \Theta_{2} \mid \Omega_{i}\right)-\Lambda\left(\Theta_{1}, \Theta_{2} \mid \Omega_{i-1}\right)}{\Lambda\left(\Theta_{1}, \Theta_{2} \mid \Omega_{N}\right)} .
\end{aligned}
$$

Then, substituting (7),

$$
p_{i}=\frac{\int_{\Omega_{0}}^{\Omega_{i}} \vartheta\left(\epsilon \mid \Theta_{1}\right) U\left(\Omega_{N}-\epsilon \mid \Theta_{2}\right) \mathrm{d} \epsilon-\int_{\Omega_{0}}^{\Omega_{i-1}} \vartheta\left(\epsilon \mid \Theta_{1}\right) U\left(\Omega_{N}-\epsilon \mid \Theta_{2}\right) \mathrm{d} \epsilon}{\int_{\Omega_{0}}^{\Omega_{N}} \vartheta\left(\epsilon \mid \Theta_{1}\right) U\left(\Omega_{N}-\epsilon \mid \Theta_{2}\right) \mathrm{d} \epsilon} .
$$

Since $R_{1}, R_{2}, R_{3} \ldots R_{N}$ are mutually exclusive events, we assume they follow multinomial distribution with cell probabilities $p_{1}, p_{2}, p_{3}, \ldots, p_{N}$. Here $\sum p_{i}=1$. Then the likelihood for these probabilities is given by

$$
L\left(\Theta_{1}, \Theta_{2} \mid p_{i}\right)=\prod_{i=1}^{N} p_{i}^{R_{i}}
$$

Fixing a plausible range of infection density parameter $\Theta_{1}$ values and from the reported $R_{1}, R_{2}, R_{3} \ldots R_{N}$, we estimate parameters of the incubation distribution $\Theta_{2}$ (say $\hat{\Theta}_{2}$ ) from (10) through the method of maximum likelihood estimation. Now, with the $\hat{\Theta}_{2}$ estimated from previous computation, along with the unknown $\Theta_{1}$ and from the reported $R_{1}, R_{2}, R_{3} \ldots R_{N}$, we estimate $\hat{\Theta}_{1}$ (an estimate of $\Theta_{1}$ ) with the likelihood function (10). $\hat{\Theta}_{1}$ is used to estimate initial values for $X_{1}$ and $Y_{1}$ to begin the epidemic predictions from calendar year $\Omega_{N}$. $\hat{\Theta}_{2}$ is used in calculating average incubation periods $1 / \eta_{1}$ and $1 / \eta_{2}$. Assuming Poisson distribution also, we can construct the above type of likelihood. However, when infection numbers are large then multinomial distribution is preferred to Poisson. We show below three sets of likelihood functions for different parametric assumptions on $V$ and $U$. 
3.1 Case $(i): V \sim$ exp. growth $(\sigma)$ and $U \sim \operatorname{Logistic}(\alpha, \gamma)$

Since $V$ has exponential growth (a common assumption for an epidemic which grows rapidly at the beginning) with parameter $\sigma$ and $U$ is a $G(\alpha, \gamma)$ variate ( $G$ is logistic, say), we have

$$
\begin{aligned}
\vartheta_{V}(\epsilon) & =e^{\sigma \epsilon} \quad \text { for all } \quad \sigma>0, \\
G_{U}(u) & =1 /\left[1+\exp ^{\left\{-\left(\frac{u-\alpha}{\gamma}\right)\right\}}\right] \text { for all } \gamma>0 .
\end{aligned}
$$

Then from (9) the likelihood equation is given by

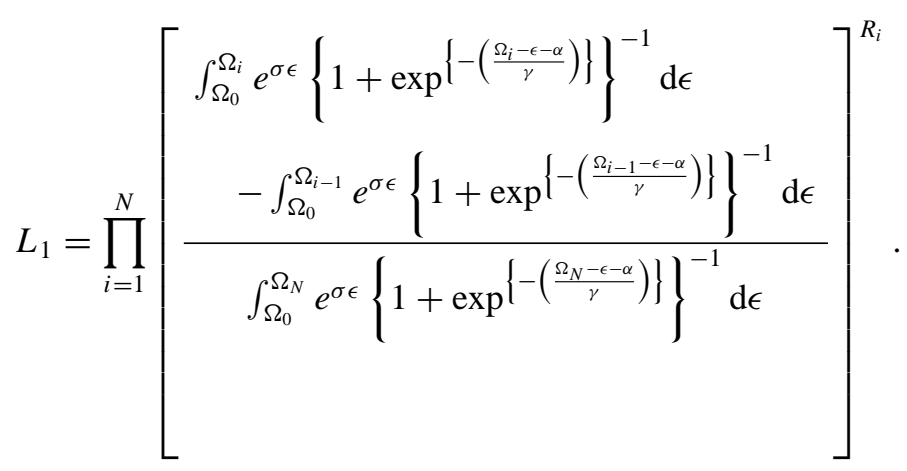

3.2 Case (ii): $V \sim$ exp.growth $(\sigma)$ and $U \sim \operatorname{Weibull}(\pi, \delta)$

Since $V$ has exponential growth as in case (i) with parameter $\sigma$ and $U$ is a $W(\pi, \delta)$ variate ( $W$ is Weibull, say), we have

$$
\begin{gathered}
\vartheta_{V}(\epsilon)=e^{\sigma \epsilon}, \text { for all } \sigma>0, \\
W_{U}(u)=1-\exp ^{\left\{-(u / \pi)^{\delta}\right\}}, \text { for all } \pi, \delta>0 .
\end{gathered}
$$

Then from (9) the likelihood equation is given by

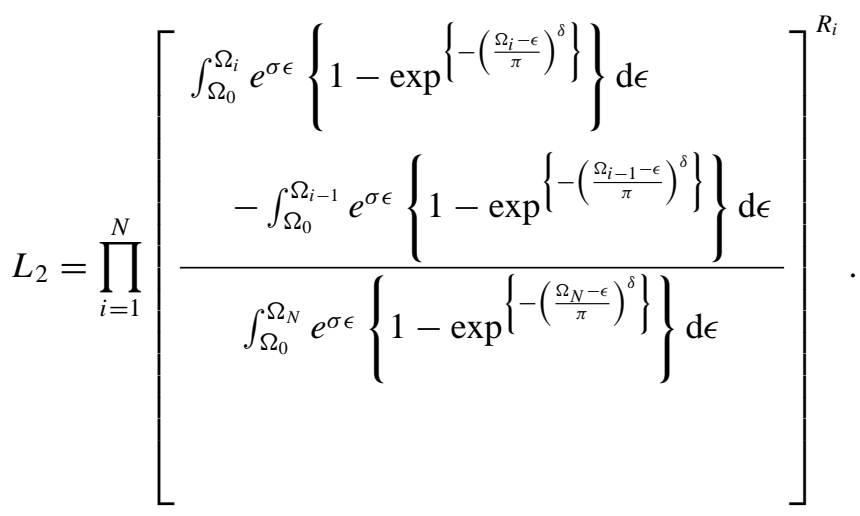




\subsection{Case (iii): $V \sim$ exp.growth $(\sigma)$ and $U \sim \operatorname{gamma}(\xi)$}

Since $V$ has an exponential growth as in cases (i) and (ii) with parameter $\sigma$ and $U$ is a $\Gamma(\xi)$ variate, we have

$$
\begin{aligned}
& \vartheta_{V}(\epsilon)=e^{\sigma \epsilon}, \quad \text { for all } \sigma>0, \\
& \Gamma_{U}(u)=\int_{0}^{u} \mathrm{e}^{-u} u^{\xi-1} \mathrm{~d} u, \quad \text { for all } \xi, u>0 .
\end{aligned}
$$

Then from (9) the likelihood is given by

$$
\begin{aligned}
L_{3}= & \prod_{i=1}^{N}\left[\frac{\int_{\Omega_{0}}^{\Omega_{i}} e^{\sigma \epsilon}\left\{\int_{\Omega_{0}}^{\Omega_{i}} e^{-\left(\Omega_{i}-\epsilon\right)}\left(\Omega_{i}-\epsilon\right)^{\xi-1} d \Omega_{i}\right\} \mathrm{d} \epsilon}{\int_{\Omega_{0}}^{\Omega_{N}} e^{\sigma \epsilon}\left\{\int_{\Omega_{0}}^{\Omega_{N}} e^{-\left(\Omega_{N}-\epsilon\right)}\left(\Omega_{N}-\epsilon\right)^{\xi-1} d \Omega_{N}\right\} \mathrm{d} \epsilon}\right. \\
& \left.-\frac{\int_{\Omega_{0}}^{\Omega_{i-1}} e^{\sigma \epsilon}\left\{\int_{\Omega_{0}}^{\Omega_{i-1}} e^{-\left(\Omega_{i-1}-\epsilon\right)}\left(\Omega_{i-1}-\epsilon\right)^{\xi-1} d \Omega_{i-1}\right\} \mathrm{d} \epsilon}{\int_{\Omega_{0}}^{\Omega_{N}} e^{\sigma \epsilon}\left\{\int_{\Omega_{0}}^{\Omega_{N}} e^{-\left(\Omega_{N}-\epsilon\right)}\left(\Omega_{N}-\epsilon\right)^{\xi-1} d \Omega_{N}\right\} \mathrm{d} \epsilon}\right] .
\end{aligned}
$$

The logistic distribution is less known for its flexible shapes when compared to the Weibull or gamma distributions. However, in bioassays logistic distribution is considered a good substitute for normal distribution. Two-parameter (scale, shape) Weibull distribution which we use here is similar to the form used by Brookmeyer \& Gail (1988). However, the theoretical properties of these parameters are the same in both the forms. The number of parameters can be increased to three to incorporate further epidemic behaviour. We (Rao \& Kakehashi 2002) have taken truncation as the third parameter and constructed the likelihood. Instead of the simple exponential, we have estimated the parametric values of the quadratic exponential. However, we did not use likelihood estimation as a supplement of parameters to a mathematical model. Considering the shape parameter to be known, statistical tests for the mean of the three-parameter Weibull was developed by Bain \& Thoman (1968). Shape parameter is crucial in epidemic forecasting and understanding the hazard of disease progression. Further details on this parameter has been discussed by (Bowman \& Shenton (2001) and Chen (1997). One can also apply the truncated logistic distribution (Tarter 1966), but the estimation procedure is more complicated. Gamma distribution also has interesting properties like the Weibull distribution. In addition, in the case of one parameter gamma $(\xi)$, the estimated value of its parameter $(\hat{\xi}$,say) is $1 / \eta$ (i.e. mean incubation period). One can also connect exponential growth rate in the early phase of the spread to maximum value of the Jacobian matrix evaluated for disease free equilibrium (Kakehashi 2000). In our next section, we give results of numerical computation for the above three cases using Indian AIDS data. Demonstration of the model equations given in $\S 2$ is carried out only for one case study which has come nearest to the epidemiological evidence. In practice all the three cases can be applied depending upon the accuracy and coverage of the data.

\section{Numerical computations}

We have taken the total number of annual AIDS cases reported to the National AIDS Control Organisation for the time period between 1986 and 2000. These numbers were divided in a 
Table 1. Estimated variables and parameters.

\begin{tabular}{|c|c|c|c|c|c|c|c|}
\hline & Cases & $\hat{\Theta}_{2}$ & $1 / \eta_{1}$ & $1 / \eta_{2}$ & $\hat{\Theta}_{1}$ & $X_{1}(' 000)$ & $Y_{1}\left({ }^{\prime} 000\right)$ \\
\hline \multirow[t]{2}{*}{ (i) } & Male & $(12 \cdot 8,0 \cdot 49)$ & $12 \cdot 8$ & - & 0.28 & 61 & - \\
\hline & Female & $(12 \cdot 1,0 \cdot 31)$ & - & $12 \cdot 1$ & 0.25 & - & 33 \\
\hline \multirow[t]{2}{*}{ (ii) } & Male & $(10 \cdot 0,10 \cdot 9)$ & $9 \cdot 5$ & - & 0.44 & 206 & - \\
\hline & Female & $(11 \cdot 6,13 \cdot 8)$ & - & $11 \cdot 3$ & 0.40 & - & 176 \\
\hline \multirow[t]{2}{*}{ (iii) } & Male & $15 \cdot 5$ & $15 \cdot 5$ & - & $0 \cdot 39$ & 633 & - \\
\hline & Female & $15 \cdot 1$ & - & $15 \cdot 1$ & 0.40 & - & 320 \\
\hline
\end{tabular}

60:40 ratio to get male and female numbers separately. (60\% of the total reported cases are males, see www.naco.nic.in). We have applied the method given in $\S 2$ using these annual numbers along with assumptions in the 3 cases discussed. Fixing $\Theta_{1}=\{0 \cdot 10-0 \cdot 60\}$ we estimated $\Theta_{2}$ and using $\hat{\Theta}_{2}$ we have fine-tuned $\Theta_{1}$. This was explained clearly in $\S 2$. $\hat{\Theta}_{1}$ values are used to get the number of HIV cases in the beginning of the year with the numerical condition that cumulative number of reported AIDS cases and estimated number match. The results of the entire estimation are given in table 1 .

\subsection{Model predictions}

The average incubation periods in case (ii) are close to the values estimated by the United Nations (2002). Estimated $X_{1}+Y_{1}$ for case (iii) ( $\simeq 1$ million) is almost one-third of the estimated figure in 2000 ( $\simeq 3.5$ millions) for India. However, for demonstration purposes of the model equations in section 1 we use the results of case (ii) from table 1 . Though the estimated HIV number for case (iii) may be closer to the National HIV estimate than those obtained from cases (i) and (ii), the lengthier incubation period (above 15 years) obtained in this case will change the dynamics. The other parameters which are not estimated are taken as follows: $\beta_{01}=0.0004, \beta_{01}^{\prime}=0.0004, C=C^{\prime}=50, r_{1(2)}=0.001, \mu=1$. These assumptions are close to the observations given by others (Anderson 1988; Kakehashi 1998, 1999; Gray et al 2001). We took reasonable withdrawal rates $\omega_{1}=10 \%, \omega_{2}=20 \%$ from the risk behaviour. These can be varied if any further survey results on adults are available. Applying all the above information to the model equations the predictions are obtained until 2011 (see figure 1).

\section{Conclusions}

Application of nonlinear differential equations model in predicting the course of epidemics is well established. The model presented here captures the dynamics of (a) virus transmission and (b) full-blown disease in males and females. Initial populations and average incubation periods are estimated through a detailed statistical approach. The parameters which could not be estimated with the available data are taken from the literature. This method of combining a mathematical model and estimating the variables, parameters is novel for epidemic prediction. All the three cases $(\S 2)$ work well (i.e. a reasonable range is obtained for estimated 


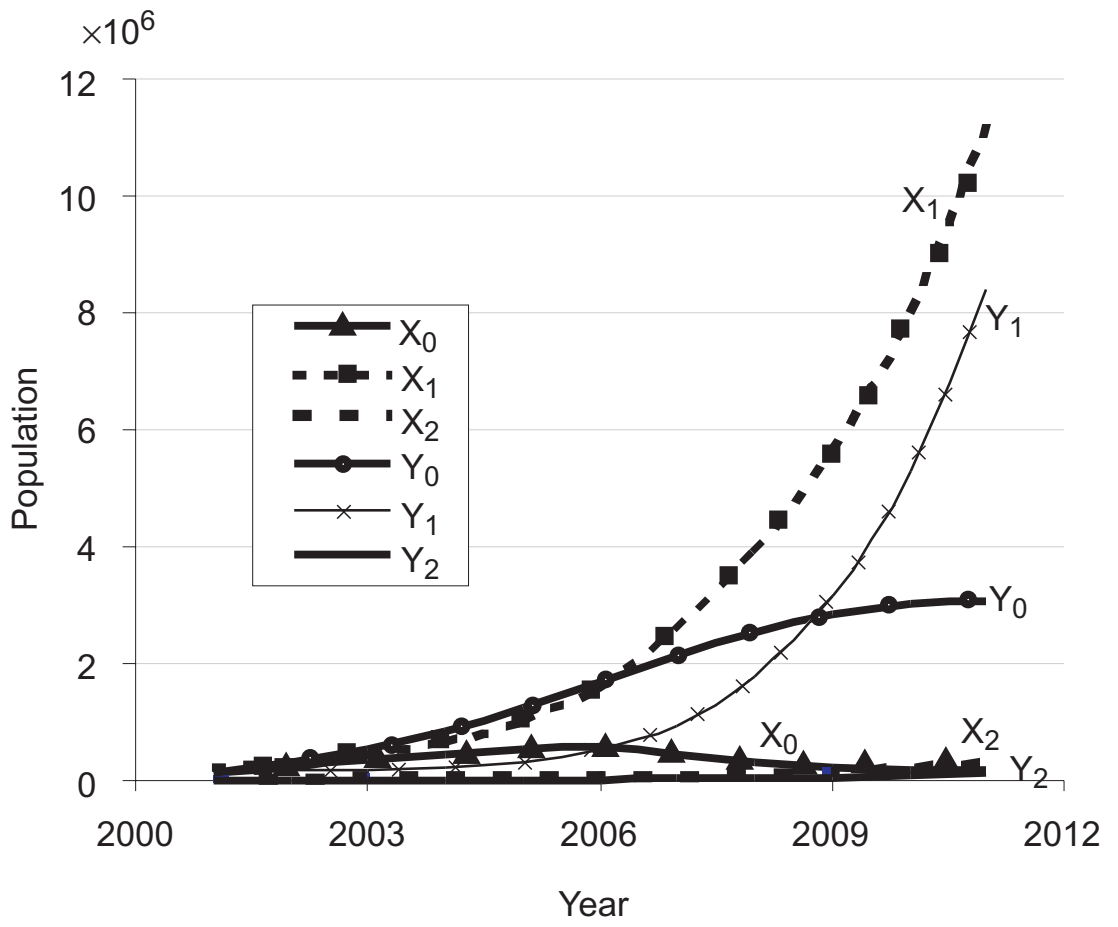

Figure 1. Trends of HIV, AIDS and susceptible population.

parameters). An advantage of the method demonstrated here is that it uses available information in addition to estimated values.

On availability of further information, one can relax the assumption of strong exponential growth in the three cases $(\$ 2)$ and can explore new information. Hence, the predictions obtained can be considered the trends of the epidemic. The model and method explained here is valid for predicting any country's data if they suits our parameter definitions. Modelling the AIDS information available in India and related technicalities can be found elsewhere (Rao 2001, 2003, Rao \& Kakehashi 2002; Rao et al 2002; Kakehashi \& Rao 2004). Incubation period in the population is highly variable. This period could vary from a couple of months to several years. Though the gamma function can mimic several exponential forms, we suggest the Weibull function as it is more suitable for modelling the incubation period owing to its highly flexible nature. Computationally Weibull is simpler if we are dealing with cumulative functions.

An unavoidable limitation at present is the under-reporting of AIDS cases used in $\S 2$. This under-reporting can be modelled as a uniform or some suitable parametric form considering that it decreases from the early stage of the epidemic to the present time period. A non-parametric form or mixture of two parametric forms cannot be ruled out. Generally under-reporting in the past is difficult to trace unless a specific study was conducted. In such cases, a gross under-reporting function $(\phi(y)$, say) can be used as a multiplier to the number of AIDS cases as an ad hoc procedure (see appendix A). However, the model and the method explained in $\S 2$ and $\S 3$ remain applicable. 


\section{Appendix A}

\section{A1. Incubation period as a multivariate logistic variable}

One can construct the likelihood equation as given in section 2 by using multivariate logistic distribution. The theoretical form (A1) for $\kappa$-variate (say) is derived by Malik \& Abraham (1973). They have not given any example. However, this form can be explored to fit multivariate incubation period data. Let $U_{i}(\mathrm{i}=1,2, \ldots \kappa)$ be a logistic random variable representing incubation period for the $i^{\text {th }}$ region in the world or within the country. Then the joint distribution $J$ of $U_{i}(\mathrm{i}=1,2, \ldots \kappa)$ can be given as

$$
J=1 /\left(1+\sum_{i=1}^{\kappa} e^{-u_{i}}\right), \quad \text { for }-\infty<u_{i}<\infty .
$$

Using (A1) we can construct likelihood function as explained in $\S 2$.

\section{A2. Adjustment of under-reporting}

There is an ad hoc procedure proposed for adjusting under-reporting of general epidemic cases and some results have been proved (Rao 2004). Let $R_{1}, R_{2}, R_{3} \ldots R_{N}$ and $T_{1}, T_{2}, T_{3}, \ldots, T_{N}$ be the numbers of reported and total AIDS cases in the calendar time intervals $\left[\Omega_{0}, \Omega_{1}\right),\left[\Omega_{1}, \Omega_{2}\right),\left[\Omega_{2}, \Omega_{3}\right), \ldots,\left[\Omega_{N-1}, \Omega_{N}\right) . \Omega_{N}$ is usually the current calendar year or previous to the current calander year. Other terminology used in $\S 2$ remains the same. Let $y_{j}(j=1,2,3, \ldots N)$ be the estimated percentage of AIDS cases that were not reported for the year $j$. Let us presume $y_{j}$ is obtained from a clinical set-up or from a survey. Then

$$
T_{i}=R_{i} / \phi\left(y_{i}\right), \quad \text { for all } \quad i \leq N \quad\left(\text { where } \phi\left(y_{i}\right)=1-y_{i}\right) .
$$

If $\phi\left(y_{i}\right)$ is a constant in (A2), then $R \propto T$ or $R=C T$, where $C$ is proportionality constant $(=\phi(y))$.

Remark 1. As $\phi(y) \rightarrow 1$, it implies $R \rightarrow T$. An important generalization is proved by the following theorem.

Theorem. Let $R, T \in \mathbf{R}^{+}-\{0\}$. For every $\alpha$-neighbourhood $N_{\alpha}(T)$ of $T$, there exist a $\delta$-neighbourhood $N_{\delta}(1)$ of 1 , around 1 , with the property that for all $\phi_{n}(y) \in N_{\delta}(1)(\phi(y) \neq$ $1)$, it follows that $R_{n} \in N_{\alpha}\left(T_{n}\right)$. Proof of this theorem is given elsewhere (Rao 2004).

We thank the anonymous referees for their comments which helped us in bringing out this final version. ASRSR thanks the Department of Science and Technology, New Delhi for financial support.

\section{References}

Anderson R M 1988 The epidemiology of HIV infection: variable incubation plus infectious periods and heterogeneity in sexual activity. J. R. Stat. Soc. A151: 66-98

Anderson R M, May R M 1991 Infectious diseases of humans: Dynamics and control (London: Oxford University Press)

Bain L J, Thoman D R 1968 Some tests of hypotheses concerning the three-parameter Weibull distribution. J. Am. Stat. Assoc. 63: 853-860 
Bowman K O, Shenton L R 2001 Weibull distributions when the shape parameter is defined. Comput. Stat. Data Anal. 36: 299-310

Brookmeyer R, Gail M H 1988 A method for obtaining short-term projections and lower bounds on the size of the AIDS epidemic. J. Am. Stat. Assoc. 83: 301-308

Chen Z 1997 Statistical inference about the shape parameter of the Weibull distribution. Stat. Prob. Lett. 36: 85-90

Gray R H et al 2001 Probability of HIV-1 transmission per coital act in monogamous, heterosexual HIV-1-discordant couples in Rakai, Uganda. Lancet 357: 1149-1153.

Kakehashi M 1998 A mathematical analysis of the spread of HIV/AIDS in Japan IMA. J Math. Appl. Med. 15: 299-311

Kakehashi M 1999 A mathematical analysis of the spread of HIV/AIDS in Japan IMA. J.Math. Appl. Med. 16: 111-112

Kakehashi M 2000 Validity of simple pair formation model for HIV spread with realistic parameter setting. Math. Pop. Stud. 8: 279-292

Kakehashi M, Rao A S R S 2004 Mathematical and statistical approaches to risk management for the prevention of HIV/AIDS and other infectious diseases. J. Med. Safety (in press)

Malik H J, Abraham B 1973 Multivariate logistic distributions. Ann. Stat. 1: 588-590

Murray J D 1989 Mathematical biology (Berlin: Springer-Verlag)

Rao A S R S 2001 A methodology to estimate incubation distribution of AIDS in delayed surveillance and censored data. Int. Stat. Conf. on Environment and Pollution (Tokyo: The Biometric Society of Japan) pp 163-167

Rao A S R S 2003a Mathematical modelling of AIDS epidemic in India. Curr. Sci. 84: 1191-1197

Rao A S R S 2003b Can we obtain realistic HIV/AIDS estimates for Inida? J Biosci, 28, 4: 367-369

Rao A S R S 2004 Limiting theorems on 'Case' reporting. Appl. Math. Lett. (in press)

Rao A S R S and Kakehashi M 2002 Incubation time distribution in back-calculation applied to HIV/AIDS in India. Indian J. Pure Appl. Math. (submitted)

Rao C N and Srivenkataramana T 2001 Projection of HIV infections in India: An alternative to backcalculation. Curr. Sci. 81: 1302-1307

Rao A S R S, Basu S, Basu A, Ghosh J K 2002 Parametric models for incubation distribution in presence of left and right censoring. Indian J. Pure Appl. Math. (submitted)

Tarter ME 1966 Exact moments and product moments of the order statistics from the truncated logistic distribution. J. Am. Stat. Assoc. 61: 514-525

United Nations 2002 The UNAIDS Reference Group on Estimates, Modelling and Projections. Improved methods and assumptions for estimation of the HIV/AIDS epidemic and its impact: Recommendations of the UNAIDS Reference Group on Estimates, Modelling and Projections. AIDS 16 (9): W1-14 